Claremont Colleges

Scholarship@ Claremont

All HMC Faculty Publications and Research

HMC Faculty Scholarship

1-1-1989

\title{
A Theoretical Study of Models for X2Y2 Zintl Ions
}

\author{
Robert J. Cave \\ Harvey Mudd College \\ Ernest R. Davidson \\ Indiana University - Bloomington \\ Philippe Sautet \\ Laboratoire de Chimie Théorique \\ Enric Canadell \\ Laboratoire de Chimie Théorique \\ Odile Eisenstein \\ Laboratoire de Chimie Théorique
}

\section{Recommended Citation}

Cave, R.J.; Davidson, E.R.; Sautet, P.; Canadell, E.; Eisenstein, E. “A Theoretical Study of Models for X2Y2 Zintl Ions,” J. Am. Chem. Soc. $1989,111,8105$. DOI: $10.1021 /$ ja00203a006

This Article is brought to you for free and open access by the HMC Faculty Scholarship at Scholarship @ Claremont. It has been accepted for inclusion in All HMC Faculty Publications and Research by an authorized administrator of Scholarship @ Claremont. For more information, please contact scholarship@cuc.claremont.edu. 
observed incompleteness in the electrochemical recovery of the deposited molecules. Cyclic voltammetry done with multilayercovered electrodes in the HT experiments suggests that the electron transfer from the additional $\mathrm{Ru}(\mathrm{bpy})_{3}{ }^{2+}$ head-group layers to the electrode surface across a single hydrocarbon chain may be possible but becomes slower as the number of the deposited layers are increased. The $\mathrm{CV}$ results obtained, however, did not exclude the possibility that the electron transfer from the $\mathrm{Ru}(\mathrm{bpy})_{2}\left(\mathrm{bpy}-\mathrm{C}_{19}\right)^{2+}$ head-group layer to the electrode and between the separated redox layers occurs via the mediation of molecular packing defects. The cyclic voltammetry performed in-trough by the HT method was also shown useful in diagnosing the physical and chemical changes of the spread surfactant molecules that occurred while they were being manipulated on trough. The monolayer coatings of $\mathrm{Ru}$ (bpy) ${ }_{2}\left(\text { bpy-C } \mathrm{C}_{19}\right)^{2+}$ at the electrode were found to have a partial blocking effect on the mass transfer of the solution species. The blocking was more significant at higher scan rates and with thicker surfactant layers and it was not very sensitive to the charges carried by the solution species.

Acknowledgment. The support of this research by the Robert A. Welch Foundation (F-079) is gratefully acknowledged.

\title{
A Theoretical Study of Models for $\mathrm{X}_{2} \mathrm{Y}_{2}$ Zintl Ions
}

\author{
Robert J. Cave, ${ }^{\dagger}$ Ernest R. Davidson, ${ }^{*, \dagger}{ }^{\dagger}$ Philippe Sautet,${ }^{\ddagger}$ Enric Canadell, ${ }^{\ddagger}$ and \\ Odile Eisenstein ${ }^{*, \ddagger}$
}

Contribution from the Department of Chemistry, Indiana University, Bloomington, Indiana 47405, and the Laboratoire de Chimie Theorique, ${ }^{\S}$ Batiment 490 , Centre de Paris-Sud, 91405 Orsay, France. Received February 23, 1989

\begin{abstract}
Ab}$ initio and extended Hückel calculations have been used to discuss the bonding scheme in $\mathrm{X}_{2} \mathrm{Y}_{2}$ neutral and ionic main group clusters. A qualitative analysis suggests that two different electron counts, 20 and 22 , are possible for the butterfly structures of these systems. This results from two orbital crossings in the correlation diagram for the tetrahedral $\left(T_{d}\right) \rightarrow$ butterfly $\left(C_{2 v}\right) \rightarrow$ square-planar $\left(D_{2 h}\right)$ transformation. Detailed ab initio computations substantiate this analysis and show that the 20 -electron butterfly structure becomes increasingly favored over the tetrahedral one in $\mathrm{X}_{2} \mathrm{Y}_{2}$ clusters when the 2 atoms have increasing electronegativity difference. These results are in agreement with the known structures for the $\mathrm{Pb}_{2} \mathrm{Sb}_{2}{ }^{2-}$ and $\mathrm{Sb}_{2} \mathrm{Bi}_{2}{ }^{2-}$ clusters (tetrahedral-like) and the $\mathrm{Tl}_{2} \mathrm{Te}_{2}{ }^{2-}$ one (butterfly-like).
\end{abstract}

\section{Introduction}

There has been continuous interest in understanding the geometry of clusters. Electron-counting rules known as the skeletal electron pair rules (SEP rules), introduced by Wade ${ }^{1}$ and Mingos, ${ }^{2}$ have been a major tool in that field, since they relate, in a simple fashion, the number of electrons in the cluster to its geometry. Originally designed to account for the structures of boranes, carboranes, and metal carbonyl clusters, where the rules have met almost total success, numerous extensions to other clusters, where the chemical nature of the vertices is different, have been possible. Extensions of the rules have also dealt with fused clusters. ${ }^{3}$ This wide success finds its support in the isolobal analogy, ${ }^{4}$ according to which specific organic and organometallic groups may be found indifferently as vertices of isostructural clusters. However, with the increasing number of synthesized clusters, exceptions have also appeared, and while these exceptions cast no doubt on the importance of the SEP rules, they clearly indicate that a range of limited validity should be considered. We have thus undertaken a theoretical study of isoelectronic clusters that either agree or disagree with predictions of the SEP rules in order to pin down the reasons for the possible breakdown of these rules in four vertex clusters.

Zintl anions ${ }^{5}$ provide an excellent target for such a study since some small main group clusters are amenable to high-level calculations as well as to qualitative bonding analysis. Our choice went to the tetratomic $Z$ intl anions $\mathrm{X}_{2} \mathrm{Y}_{2}{ }^{2-}$ with a total number of 20 electrons. The SEP rules suggest that a tetranuclear cluster with a total number of 20 electrons should be tetrahedral $\left(\mathrm{P}_{4}\right.$ for instance). This is a nido cluster according to the terminology of

\footnotetext{
* Authors to whom correspondence should be addressed.

+ Indiana University.

¿aboratoire de Chimie Theorique.

Associated with the CNRS (UA 506) and is a member of the Institut de Chimie Molêculaire d'Orsay and Institute de Physico-Chimie Molêculaire.
}

Wade and Mingos. This is in fact the case for ${ }^{6,7} \mathrm{~Pb}_{2} \mathrm{Sb}_{2}{ }^{2-}$ and $\mathrm{Sn}_{2} \mathrm{Bi}_{2}{ }^{2-}$ but not for ${ }^{8} \mathrm{Tl}_{2} \mathrm{Te}_{2}{ }^{2-}$, which takes a butterfly shape with $\mathrm{Te}$ at the wing tip, $\mathrm{Tl}$ at the back bone, and a large angle between the two wings. ${ }^{9}$

The present calculations were undertaken to obtain qualitative and semiquantitative information regarding these ions. A series of neutral and anionic model compounds of the form $\mathrm{X}_{2} \mathrm{Y}_{2}$ have been examined to assess whether any underlying trends can be observed in the structures or energetics of these molecules, based on the results of ab initio and extended Hückel calculations. Other theoretical studies have appeared for the "Zintl" ions and systems similar to them, ${ }^{10-14}$ and the relationship of the electronegativity difference between the atoms in the molecule and the molecular geometry has also been explored with relativistic extended Hückel

(1) Wade, K. J. Chem. Soc., Chem. Commun. 1971, 792. Wade, K. Adv. Inorg. Chem. Radiochem. 1976, 18, 1 .

(2) Mingos, D. M. P. Nature 1972,236,99, Mingos, D. M. P. Acc. Chem Res. 1984, 17, 311. Mingos, D. M. P.; Johnston, R. L. Struct. Bonding (Berlin) 1987, 68, 29.

(3) Mingos, D. M. P. J. Chem. Soc., Chem. Commun. 1983, 706

(4) Hoffmann, R. Angew. Chem., Int. Ed. Engl. 1982, 21, 711.

(5) Schäfer, H.; Eisenmann, B.; Müller, V. Angew. Chem., Int. Ed. Engl. 1973, 12, 694

(6) Critchlow, S. C.; Corbett, J. D. Inorg. Chem. 1985, 24, 979.

(7) Critchlow, S. C. Corbett, J. D. Inorg. Chem. 1982, 21, 3286.

(8) Burns, R. C.; Corbett, J. D. J. Am. Chem Soc. 1981, 103, 2627

(9) For a complete survey, see: Corbett, J. D. Chem. Rev. 1985, 85, 383.

(10) Rothman, M. J.; Bartell, L. S.; Ewig, C. S.; Van Wazer, J. R. J. Comput. Chem. 1980, 1,64 .

(11) Rothman, M. J.; Bartell, L. S.; Lohr, L. L., Jr. J. Am. Chem. Soc. 1981, 103, 2482 .

(12) Lohr, L. L., Jr. Inorg. Chem. 1981, 20, 4229.

(13) Savin, A.: Vogel, K.; Preuss, H.; Stoll, H.; Nesper, R. von Schnering,

H.-G. J. Am. Chem. Soc. 1988, 110, 373.

(14) Axe, F. U.; Marynick, D. S. Inorg. Chem. 1988, 27, 1426.

(15) Lohr, L. L., Jr. Int. J. Quantum Chem. 1984, 25, 211. 
theory. ${ }^{15}$ In this study, we have neglected counterions when examining anions. This neglect will generally destabilize the anions relative to the separated atom limit, but we assume that it has no qualitative effect on the relative energies of different conformations of the $X_{2} Y_{2}$ molecule.

While the Zintl compounds are generally anions, we have concentrated on a set of model neutral molecules that are similar to the Zintl ions. There are several reasons for this. First, anions are notoriously difficult problems for ab initio methods. Thus, the calculations on the neutral molecules provide a consistency check on the calculations for the anions. Second, the neutral species are of interest in their own right. While they have yet to be synthesized, it is nevertheless of interest to examine their properties and assess their stabilities. Third, the neutral compounds provide a wider range of electronegativity differences. Since we wish to examine how the electronegativity difference of $\mathrm{X}$ and $\mathrm{Y}$ relates to the most stable structure, it is useful to examine systems for which this difference is as great as possible.

\section{Computational Methods}

The $a b$ initio calculations were based on the relativistic pseudopotentials of Wadt and Hay. ${ }^{16}$ Use of these pseudopotentials reduces the size of the computational problem and also incorporates the dominant oneelectron relativistic effects that would be neglected in an all-electron nonrelativistic calculation. Given the high atomic number of the atoms examined, it is expected that neglect of relativistic contractions of the atomic radii could lead to qualitative errors in the bond lengths of these molecules.

We used the $(3 s, 3 p)$ basis sets of Wadt and Hay ${ }^{16}$ contracted to $(2 s, 2 p)$ using the contraction coefficients suggested there. For the geometry optimizations reported below that are in $C_{1}$ symmetry, this basis was used with no further additions. For calculations in $C_{2 v}$ symmetry, the atomic basis sets were augmented with one set of d polarization functions on each atom. The exponents of these functions were as follows: Sn, 0.12; Sb, 0.15; Te, 0.1833; I, 0.20; Tl, 0.10; Pb, 0.11; Bi, 0.13 . The calculated energies and equilibrium geometries are relatively insensitive to the specific values of these exponents. For the calculations on neutral (but not anionic) species in $C_{20}$ symmetry, we further augmented these basis sets with extra $s$ and $p$ functions which were more diffuse than the original $3 \mathrm{~s}$ or $3 \mathrm{p}$ functions. The exponents for these (sp) functions were as follows: Sb, $(0.02707,0.02705)$; Te, $(0.03361,0.0301)$; I, $(0.0384,0.0349) ; \mathrm{Tl},(0.02178,0.0136) ; \mathrm{Pb},(0.02374,0.01942) ; \mathrm{Bi}$, $(0.02863,0.02533)$. No neutral molecules containing $\mathrm{Sn}$ were examined, so no diffuse $s$ and $p$ functions are reported for it. Thus, for the neutral molecules in $C_{2 v}$ symmetry, the total basis set on each atom was (4s $4 \mathrm{pld} / 3 \mathrm{~s} 3 \mathrm{pld}$ ) except where noted. For the calculations on the anions, the basis set on each atom was $(3 \mathrm{~s} 3 \mathrm{p} 1 \mathrm{~d} / 2 \mathrm{~s} 2 \mathrm{pld})$.

Unless otherwise noted, we assume $C_{20}$ symmetry. Below we refer to the general compound of this type as $\mathrm{X}_{2} \mathrm{Y}_{2}$, with $\mathrm{X}$ being $\mathrm{Tl}, \mathrm{Pb}$, or $\mathrm{Bi}$ and $\mathrm{Y}$ being $\mathrm{Sn}, \mathrm{Sb}, \mathrm{Te}$, or $\mathrm{I}$. In $C_{2 v}$ symmetry, the $\mathrm{X}$ atoms are along the $x$ axis (with possible nonzero $z$ coordinates) and the $Y$ atoms are placed along the $y$ axis (again, having possible nonzero $z$ coordinates as shown in structure 1). The $z$ axis is thus the $C_{2}$ axis, and the $x z$ and $y z$ planes are the mirror planes for the molecule. When $C_{2 v}$ symmetry is imposed, all $X-Y$ bond lengths are equivalent, and therefore only rhomboidal distortions (and not rectangular distortions) are possible.
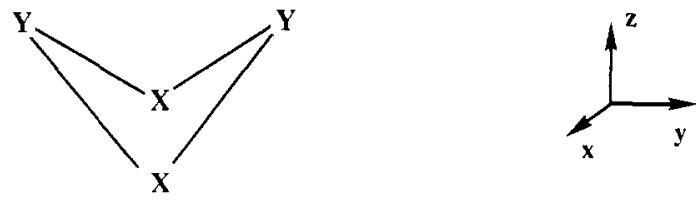

1

All geometry optimizations were performed with HONDO. ${ }^{17}$ The single-point SCF calculations were obtained with MELDF, ${ }^{18}$ The SCF results are from spin-restricted open- and closed-shell Hartree-Fock calculations. The atomic calculations were performed in $C_{2 v}$ symmetry

(16) Wadt, W. R.; Hay, P. J. J. Chem. Phys. 1985, 82, 284.

(17) The geometry optimizations were performed with HONDO, written by M. Dupuis, 1985 .

(18) The MELDF series of electronic structure codes was developed by $L$. E. McMurchie, S. T. Elbert, S. R. Langhoff, E. R. Davidson, and D. Feller, and was extensively modified by D. C. Rawlings.

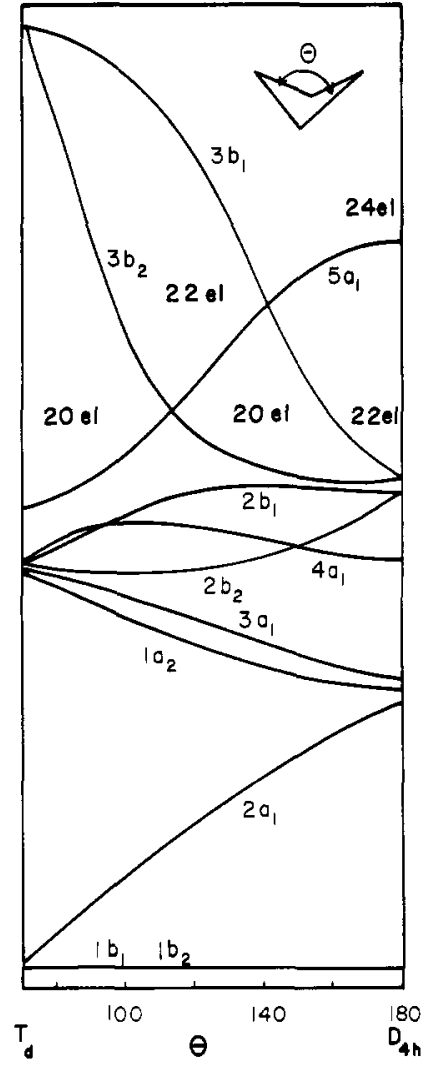

Figure 1. Walsh diagram for the transformation shown in Scheme I for $X_{4}$. The lowest occupied $a_{1}$ orbital energy has been omitted from the diagram. The empty orbital degenerate with $3 b_{1}$ and $3 b_{2}$ at the $T_{d}$ geometry has also been omitted.

\section{Scheme I}

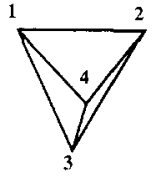

$T_{d}$

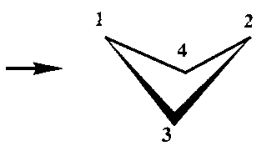

$\mathrm{C}_{2 \mathrm{r}}$
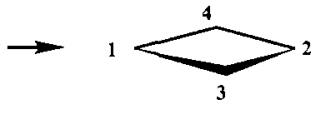

$D_{4 h}$ but were not symmetry equivalenced, to allow direct comparison with the molecular energies. The energy difference between the symmetry equivalenced and inequivalenced atomic results is expected to be less than 0.001 hartree in all cases.

In calculations on anions, we have not augmented the atomic basis sets with the more diffuse $s$ and $p$ functions given above. Had we done so, the added electrons would have mainly occupied highly diffuse orbitals, rather than being in orbitals localized near the molecule. Since the actual anionic systems possess nearby (large) counterions, a stabilization and localization of the additional charges is expected. Thus, by limiting the basis in this way, we impose localization on the added electrons. While this is somewhat artificial, we assume that it is adequate for relative energies of the molecules of interest. Population analyses and net atomic charges are based on the Mulliken population analysis.

For most compounds examined below, we find two local stationary points of $C_{2 w}$ symmetry, one nearly tetrahedral (Td), the other nearly square planar (SP). In one case, that of $\mathrm{Pb}_{2} \mathrm{Te}_{2}$, we study a $C_{2 v}$ linear reaction path between these two stationary points. In $C_{20}$ symmetry, only three independent coordinates exist for the $X_{2} Y_{2}$ compounds, and we choose them as the XYX angle, the YXY angle, and the $X Y$ bond length. Geometries intermediate between the two stationary points are parameterized as

$$
R(q)=R_{1}+q\left(R_{2}-R_{1}\right)
$$

where $R_{1}$ and $R_{2}$ are the initial and final values of the given coordinate, and $q$, the "reaction parameter", varies from 0 (Td) to 1 (SP). It should be noted that this is not a true reaction path ${ }^{19}$ but rather an approximation to it based on the assumption that the path is one of least motion.

(19) Fukui, K. Acc. Chem. Res. 1981, 14, 363. 


\section{Qualitative Description of the Bonding Scheme}

The Walsh diagram, obtained by means of $\mathrm{EHT}^{20}$ calculations for the tetrahedral $\left(T_{d}\right) \rightarrow$ butterfly $\left(C_{2 v}\right) \rightarrow$ square-planar $\left(D_{2 h}\right)$ (Scheme I) transformation of $\mathrm{X}_{4}$ is shown in Figure 1. The angle, $\theta$, between the two wings varying from $70.5^{\circ}$ to $180^{\circ}$ is used as the reaction coordinate. Along with the wing flap, the bond distance between the two atoms that will become the backbone of the butterfly is allowed to stretch in a stepwise manner to allow a smooth transformation of the molecular orbitals along the path. The amount of backbone stretching associated with the wing flipping is somewhat arbitrary, but the main features of the Walsh diagram are not greatly affected by the quantitative aspect of this correlation.

The orbitals of Figure 1 have been labeled within the $C_{2 v}$ symmetry point group which is maintained for the whole path. The occupancy for 20 electrons in the $T \mathrm{~d}$ structure is thus $\left(1 a_{1}\right)^{2}\left(1 b_{2}\right)^{2}\left(1 b_{1}\right)^{2}\left(2 a_{1}\right)^{2}\left(1 a_{2}\right)^{2}\left(3 a_{1}\right)^{2}\left(2 b_{2}\right)^{2}\left(2 b_{1}\right)^{2}\left(4 a_{1}\right)^{2}\left(5 a_{1}\right)^{2}$. It corresponds to the filling of all lone pair and bonding orbitals until a large gap is reached, and it thus accounts for the stability of the structure with this electron count. The two lowest empty orbitals that are going to have a determinant role in the $T_{d} \rightarrow$ $C_{2 v} \rightarrow D_{4 h}$ transformation are shown as 2 and 3 . For a perfect $T_{d}$ or $D_{4 h}$ geometry in an homoatomic system, $2\left(3 \mathrm{~b}_{2}\right)$ and $\mathbf{3}\left(3 \mathrm{~b}_{1}\right)$ are degenerate in energy. $\mathbf{2}$ is strongly antibonding between the

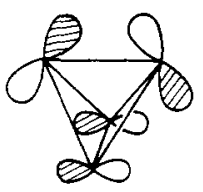

2

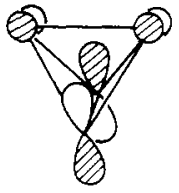

3

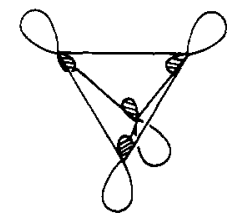

4 two $\mathrm{Y}$ atoms which are going to become the wing tip while $\mathbf{3}$ is strongly antibonding between the two $\mathrm{X}$ atoms which are going to form the backbone of the butterfly.

Since the reaction coordinate stretches the $Y-Y$ bond faster than it stretches the $X-X$ bond, $3 b_{2}$ goes down in energy faster than $3 b_{1}$. These two orbitals correlate with the nonbonding degenerate $\pi$ orbitals of $D_{4 h}$. At the same time, $4\left(5 a_{1}\right)$, which is a wholly in-phase orbital that correlates with the antibonding $\pi$ orbital of $D_{4 h}$, is raised in energy, so that two orbital crossings occur along the reaction path, first with $3 \mathrm{~b}_{2}$ and then second with $3 b_{1}$. Assuming that a stable electron occupancy attempts to put electrons in all bonding or weakly bonding orbitals until a reasonable HOMO-LUMO gap is reached, it is apparent that two electron counts are plausible for the butterfly cluster, either 20 electrons $\left(3 b_{2}\right)^{2}\left(5 a_{1}\right)^{0}$ for $\theta$ around $150^{\circ}$ or 22 electrons $\left(3 b_{2}\right)^{2}$ $\left(5 a_{1}\right)^{2}\left(3 b_{1}\right)^{0}$ for $\theta$ around $120^{\circ}$. The 22 -electron count is the one suggested by the SEP rule. It corresponds to the addition of two electrons to a tetrahedron that according to the displayed Walsh diagram cleaves one bond ${ }^{21}$ (occupancy of $3 b_{2}$ ) -i.e., the one between the wing tip atoms-while it maintains a bond between the backbone atoms. $3 b_{1}$ should remain empty and at high energy ensure a large HOMO-LUMO gap. Because there is no orbital crossing between occupied and empty orbitals along the $T_{d} \rightarrow C_{2 v}$ transformation for 22 electrons, the ring opening is an allowed process. ${ }^{22}$ In contrast, such a transformation is not permitted for 20 electrons $\left(5 a_{1}-3 b_{2}\right.$ crossing), so both structures, tetrahedron and butterfly, may be stable. For the 20 -electron count, the stable butterfly structure lies in a part of the reaction coordinate where the 1-2 bond is definitely broken and the 3-4 one is fairly weakened. Although the EHT calculations cannot determine in any precise manner the value of $\theta$ and that of the backbone

(20) Hoffmann, R. J. Chem. Phys. 1963, 39, 1397. A modified Wolfsberg-Helmholz formula was used to calculate the off-diagonal $H_{i j}$ element values: Ammeter, J. H.; Bürgi, H. B.; Thibeault, J.; Hoffmann, R. J. Am. Chem. Soc. 1978, 100, 3686

(21) Burdett, J. K. Molecular Shapes; Wiley: New York, 1980; Chapter

(22) Woodward, R. B.; Hoffmann, R. The Conservation of Orbital Symmetry; Academic Press: New York, 1969. stretching, the qualitative bonding picture is correctly reproduced, as will be seen from the ab initio calculations.

Additional flattening of the butterfly accompanied by further stretching of the backbone leads to the square-planar structure, where $3 b_{1}$ is now at a low energy so that the proper electron count for a closed shell corresponds to $\left(3 b_{2}\right)^{2}\left(3 b_{1}\right)^{2}$, i.e., to 22 electrons. The transformation of the 22-electron butterfly into the squareplanar is a forbidden process, so, in this case also, two types of structures may be stable for the same electron count. Different $\mathrm{X}_{4}{ }^{2+}\left(\mathrm{X}=\mathrm{S},{ }^{23} \mathrm{Se}^{24} \mathrm{Te}^{25}\right)$ and $\mathrm{X}_{4}{ }^{2-}\left(\mathrm{X}=\mathrm{Bi}^{26} \mathrm{Sb}^{27}\right)$ square-planar clusters with this electron count have been characterized. Often, such species can also be found as part of the intermetallic Zintl phases. For instance, $\mathrm{Ca}_{11} \mathrm{Bi}_{10}{ }^{28}$ contains $\mathrm{Bi}_{4}{ }^{2-}$ units, $\mathrm{Yb}_{11} \mathrm{Sb}_{10}{ }^{29}$ and $\mathrm{Ca}_{11} \mathrm{Sb}_{10}{ }^{28}$ contain $\mathrm{Sb}_{4}{ }^{2-}$, and $\mathrm{Ho}_{11} \mathrm{Ge}_{10}{ }^{30}$ contains $\mathrm{Ge}_{4}{ }^{6-}$, all of which are square planar. ${ }^{31} \mathrm{Si}_{4}{ }^{6}$, found in the intermetallic phase $\mathrm{Si}_{4} \mathrm{Ba}_{3},{ }^{32}$ is, to our knowledge, the only other butterfly main group cluster with the alternative electron count. It should be noted that a total number of 24 electrons has been suggested by the SEP rules for a square-planar arachno cluster. This would put electrons in the high-lying $5 \mathrm{a}_{1}$ orbital, which is in fact well separated from the next higher orbital. Bicyclobutane has been considered as one example of a 24-electron almost square planar arachno cluster. However, a 24-electron-count square-planar system would correspond to highly anionic compounds in main group chemistry and may not be stable by themselves except as part of some intermetallic Zintl phase (see below).

If the $\mathrm{XX}$ and $\mathrm{YY}$ bonds were stretched equally so as to preserve $D_{2 d}$ symmetry, $3 \mathrm{~b}_{1}$ and $3 \mathrm{~b}_{2}$ would be degenerate for all $\theta$. Hence, it is clear that the existance of a 20-electron butterfly-type cluster is linked to the fact that one bond is considerably more stretched than the other. This suggests that heteroatomic $X_{2} Y_{2}$ clusters are likely candidates for such a structure and raises the question of which atom, $\mathrm{X}$ or $\mathrm{Y}$, is going to be at the wing tip or at the backbone of the butterfly. As indicated by Corbett, a localized Lewis structure for the butterfly cluster shows that the wing tip atoms have more lone pairs than the backbone atoms. Our analysis lead to a similar conclusion. In an heteroatomic $\mathrm{X}_{2} \mathrm{Y}_{2}$ cluster, the $3 b_{2}$ and $3 b_{1}$ orbitals are no longer degenerate. The antibonding orbital localized on the more electronegative atoms, $Y$, is at a lower energy than the antibonding one localized on the more electropositive atom, $\mathrm{X}$ for all values of $\theta$. Stretching the $\mathrm{Y}-\mathrm{Y}$ bond to a larger extent than the $\mathrm{X}-\mathrm{X}$ bond allows for earlier crossing of $3 b_{2}$ and $5 a_{1}$ for all values of $\theta$, which leads to a larger range of $\theta$ values between the two orbital crossings $\left(5 \mathrm{a}_{1}-3 \mathrm{~b}_{2}\right.$ and $\left.5 a_{1}-3 b_{1}\right)$ and hence provides greater HOMO-LUMO gaps in that zone. Since the orbital localized on the more electronegative atoms is always the one with the lowest energy (near the tetrahedral and the square-planar structures), stretching the $Y-Y$ bond can only increase the initial gap between these orbitals.

The previous description can be summarized in the following manner. The bond that is more easily reduced is the bond between the electronegative atoms, while the bond that is easier to oxidize is the one between the electropositive atoms. If one considers that a formal redox process has occurred in the molecule, the electrons have partially moved from $\sigma_{\mathrm{XX}}$ into $\sigma^{*}{ }_{\mathrm{YY}}$. Both bonds have thus

(23) Passmore, J.; Sutherland, G.; White, P. S. J. Chem. Soc., Chem. Commun. 1980, 330.

(24) Cardinal, G.; Gillespie, R. J.; Sawyer, J. F.; Vekris, J. E. J. Chem Soc., Dalton Trans. 1982, 765. 357 .

(26) Cisar, A.; Corbett, J. D. Inorg. Chem. 1977, 16, 2482

(27) Critchlow, S. C.; Corbett, J. D. Inorg. Chem. 1984, 23, 770

(28) Deller, K.; Eisenmann, B. Z. Naturforsch. B 1976, 31, 29.

(29) Clark, H. L.; Simpson, H. D.; Steifink, H. Inorg. Chem. 1970, 9, 1962.

(30) Smith, G. S.; Johnson, Q.; Tharp, A. G. Acta Crystallogr. 1967, 23,

(31) The complete charge transfer is used only as a way to count electrons-i.e., the occupied orbitals of these systems will contain as the major component the occupied orbitals of the square-planar/tetrahedral units, which corresponds to the 22-electron/20-electron count - and of course does not imply a real complete charge transfer.

(32) Eisenmann, B.; Janzon, K. H.; Schäfer, H.; Weiss, A. Z. Naturforsch. $1969,246,457$ 
Table I. Neutral and Anionic Atomic SCF Energies ${ }^{a}$

\begin{tabular}{lcc}
\hline atom & energy, hartrees & EA, kcal/mol \\
\hline & $(3 \mathrm{~s} 3 \mathrm{pld} / 2 \mathrm{~s} 2 \mathrm{pld})$ & \\
$\mathrm{Sn}$ & -3.2334 & 17.6 \\
$\mathrm{Sn}^{-}$ & -3.2614 & \\
$\mathrm{Sb}$ & -5.2600 & -14.9 \\
$\mathrm{Sb}^{-}$ & -5.2362 & 15.9 \\
$\mathrm{Te}$ & -7.8566 & \\
$\mathrm{Te}^{-}$ & -7.8819 & 50.1 \\
$\mathrm{I}$ & -11.1573 & \\
$\mathrm{I}^{-}$ & -11.2371 & \\
$\mathrm{Tl}$ & -1.9216 & \\
$\mathrm{Ti}^{-}$ & -1.9089 & 14.1 \\
$\mathrm{~Pb}^{-}$ & -3.3377 & \\
$\mathrm{~Pb}$ & -3.3601 & -16.7 \\
$\mathrm{Bi}^{-}$ & -5.3086 & \\
$\mathrm{Bi}$ & -5.2820 & \\
& $(4 \mathrm{~s} 4 \mathrm{pld} / 3 \mathrm{~s} 3 \mathrm{pld})$ & \\
$\mathrm{Sb}$ & -5.2602 & \\
$\mathrm{Te}$ & -7.8571 & \\
$\mathrm{I}$ & -11.1577 & \\
$\mathrm{Tl}$ & -1.9217 & \\
$\mathrm{~Pb}$ & -3.3379 & \\
$\mathrm{Bi}$ & -5.3087 &
\end{tabular}

${ }^{a}$ Results from open- and closed-shell restricted Hartree-Fock calculations.

been cleaved in the process. This suggests that a large electronegativity difference between the atoms in a cluster may lead to a butterfly structure that will differ from the tetrahedral one by two covalent bonds. Support for this analysis comes from the fact that $\mathrm{Tl}_{2} \mathrm{Te}_{2}{ }^{2-}$ with 20 electrons has a butterfly structure, whereas $\mathrm{Pb}_{2} \mathrm{Sb}_{2}{ }^{2-}$ and $\mathrm{Sb}_{2} \mathrm{Bi}_{2}{ }^{2-}$, with the same electron count, are tetrahedral-like. As mentioned above, $\mathrm{Si}_{4}{ }^{6-}$ is the only example of the alternative 22 -electron count for a butterfly structure in the main group elements. ${ }^{32}$ In contrast, all homoatomic 20 -electron clusters are tetrahedral. Examples of such clusters found in intermetallic phases include $\mathrm{Tl}_{4}{ }^{8-}$ found in $\mathrm{Na}_{2} \mathrm{Tl},{ }^{33} \mathrm{Si}_{4}{ }^{4-}$ and $\mathrm{Ge}_{4}{ }^{4-}$ found in numerous phases with the $\mathrm{KGe}^{34}$ or $\mathrm{BaSi}_{2}{ }^{35}$ type structures, and $\mathrm{Sn}_{4}{ }^{4-}$ in $\mathrm{KSn}^{36}$ or $\mathrm{Pb}_{4}{ }^{4-}$ in $\mathrm{NaPb}{ }^{37}$

Does the existence of two different possible electron counts for butterfly clusters result from some failure of the SEP counting rules? Not really. In fact, this double-electron count stems from the somewhat ill-defined geometry of the butterfly. It is clear from the preceding discussion that, in the real potential energy surfaces for the structural interconversions, the butterfly with the higher electron count ( 22 electrons, $5 \sigma$ bonds) will tend to keep its backbone much shorter ( $\mathrm{see}^{\mathrm{Si}}{ }_{4}{ }^{6-}$ ) than in the case of the low-electron-count alternative ( 20 electrons, $4 \sigma$ bonds; see $\left.\mathrm{Tl}_{2} \mathrm{Te}_{2}{ }^{2-}\right)$. The approach assumed by the SEP counting rules, which focuses on the tetrahedron (20 electrons, $6 \sigma$ bonds) as the basic structure, leads to only one of the possible countings ( 22 electrons, $5 \sigma$ bonds) when 2 electrons are added to $\sigma^{*} \mathrm{YY}$. The second electron counting ( 20 electrons) for the butterfly can only be reached by removing 2 electrons from the closed-shell square-planar ( 22 electrons, $4 \sigma$ bonds, $6 \pi$ electrons) reference structure. This was the approach chosen in the perceptive work by Burns and Corbett. ${ }^{8}$ Interestingly, the 22 -electron count appropriate for the butterfly cluster derived from the tetrahedron is the same electron count as that of the reference square-planar structure, whereas the 20-electron count appropriate for the butterfly cluster derived from the square-planar one is that of the reference tetrahedron. As the Walsh diagram of Figure 1 shows, this is a consequence of the double orbital crossing during the transformation $T_{d} \rightarrow C_{2 v} \rightarrow D_{2 h}$. In the next sections, we will try to put these results on a more quantitative basis by performing

(33) Smith, J. F.; Hansen, D. A. Acta Crystallogr. A. 1966, 21, 88

(34) Busmann, E. Anorg. Allg. Chem. 1961, 313, 90.

(35) Schäfer, H.; Janzon, K. H.; Weiss, A. Angew. Chem., Int. Ed. Engl. 1963, 2, 393.

(36) Hewaidy, I. F.; Bussman, E.; Klemm, W. Z. Anorg. Allg. Chem. $1964,328,283$.

(37) Marsch, R. E.; Shoemaker, D. P. Acta Crystallogr. 1953, 6, 197.
Table II. Neutral and Anionic Closed-Shell Singlet Diatomic Molecules $^{a}$

\begin{tabular}{|c|c|c|c|c|}
\hline molecule & $R_{,}^{b} \AA$ & $\begin{array}{l}\text { energy, } \\
\text { hartrees }\end{array}$ & $\begin{array}{c}\Delta E^{c} \\
\mathrm{kcal} / \mathrm{mol}\end{array}$ & charges $^{d}$ \\
\hline TII & 2.87 & -13.1825 & -64.7 & $\begin{array}{l}\mathrm{Tl}=+0.56 \\
\mathrm{I}=-0.56\end{array}$ \\
\hline $\mathrm{PbTe}$ & 2.57 & -11.2619 & -42.0 & $\begin{array}{l}\mathrm{Pb}=+0.52 \\
\mathrm{Te}=-0.52\end{array}$ \\
\hline $\mathrm{BiSb}$ & 2.50 & -10.5450 & +15.0 & $\begin{array}{l}\mathrm{Bi}=+0.17 \\
\mathrm{Sb}=-0.17\end{array}$ \\
\hline $\mathrm{TlTe}^{-}$ & 2.68 & -9.8770 & -46.1 & $\begin{array}{l}\mathrm{Tl}=-0.09 \\
\mathrm{Te}=-0.91\end{array}$ \\
\hline $\mathrm{PbSb}^{-}$ & 2.57 & -8.6344 & -9.0 & $\begin{array}{l}\mathrm{Pb}=-0.29 \\
\mathrm{Sb}=-0.71\end{array}$ \\
\hline $\mathrm{BiSn}^{-}$ & 2.58 & -8.5811 & -7.0 & $\begin{array}{l}\mathrm{Bi}=-0.59 \\
\mathrm{Sb}=-0.41\end{array}$ \\
\hline
\end{tabular}

${ }^{a}$ Results from closed-shell restricted Hartree-Fock calculations. The basis set for the neutral species is $(4 s 4 \mathrm{pld} / 3 \mathrm{~s} 3 \mathrm{pld})$. For the anions, the $(3 \mathrm{~s} 3 \mathrm{pld} / 2 \mathrm{~s} 2 \mathrm{pld})$ basis set was used. ${ }^{b}$ The optimized bond length in angstroms. 'The energy of the molecule relative to separated atoms in the appropriate basis set. The atomic energies are from Table I. ${ }^{d}$ Atomic charges based on Mulliken population analysis.

$a b$ initio calculations on several $X_{2} Y_{2}$ clusters.

\section{Ab initio Calculations for $\mathrm{X}_{2} \mathrm{Y}_{2}$ Models}

1. Computational Results. In Table I, we report the results from SCF calculations on the atoms (both neutral and anionic) examined in the present study. Results from both basis sets are presented, and it is seen that the total energies change by a quite small amount. Nevertheless, the diffuse functions and polarization functions included in the $(4 \mathrm{~s} 4 \mathrm{pld} / 3 \mathrm{~s} 3 \mathrm{pld})$ basis can be useful in describing ionic bonding, and this basis is used for the neutral $\mathrm{C}_{2 \mathrm{v}}$ $\mathrm{X}_{2} \mathrm{Y}_{2}$ compounds studied here. The SCF electron affinities are not expected to be accurate, since the correlation energies of the $N$-electron and $(N+1)$-electron systems are quite different.

In Table II, we report the results from calculations on neutral and ionic closed-shell diatomics comprised of the atoms considered in Table I. The bond lengths in Table II are the optimized lengths obtained with closed-shell Hartree-Fock wave functions. It is seen that all diatomics except $\mathrm{BiSb}$ are bound. $\mathrm{PbSb}^{-}$and $\mathrm{BiSn}^{-}$are bound only weakly at the SCF level but are expected to be more strongly bound when correlation is included. The charges presented in Table II are consistent with the differences in electronegativity between the atoms. It should be noted that Mulliken populations are dependent on the basis set used, and the actual charges should not be taken too seriously. However, they can be useful in comparison of a series of compounds using comparable basis sets, and it is in this vein that we employ them here.

In Table III, the results for a series of molecules (neutral and dianionic) of the form $\mathrm{X}_{2} \mathrm{Y}_{2}$ are shown. Along with the total energies and atomic charges, the optimized $C_{2 v}$ geometries are given. Since the Zintl anions are known to occur in either Td-like or SP-like structures ${ }^{1}$ we sought stationary points of each type for the compounds in Table III. This was accomplished by beginning the geometry optimizations near where the assumed minimum was expected. The geometries are designated as $\mathrm{Td}$ and SP in Table III. When discussing these geometries below, we refer to them as tetrahedral or square planar, with the understanding that they do not possess full $T_{d}$ or $D_{4 h}$ symmetry, but that they approximate these idealized structures.

Several interesting points can be seen for the $\mathrm{X}_{2} \mathrm{Y}_{2}$ compounds. First, for all but $\mathrm{Tl}_{2} \mathrm{I}_{2}$, two $C_{2 v}$ stationary points are found. Second, within the neutral series and within the dianion series, there is a change in which of the two structures is lowest in energy. In general, we find that the larger the electronegativity difference between the constituent atoms the more the SP structure is favored over the Td structure. This is particularly true for $\mathrm{Tl}_{2} \mathrm{I}_{2}$, where the electronegativity difference is greatest, and we find no stationary point for the Td structure. Third, for the compounds with two stationary points, the largest charge separation occurs for the SP structure.

The results from Table III are all based on single-configurational SCF calculations. Considering only the valence orbitals 
Table III. Neutral and Ionic Closed-Shell $\mathrm{X}_{2} \mathrm{Y}_{2}$ Molecules ${ }^{a}$

\begin{tabular}{|c|c|c|c|c|c|c|c|c|}
\hline & structure $^{b}$ & $\begin{array}{l}\text { energy, } \\
\text { hartrees }\end{array}$ & $\begin{array}{c}\Delta E,^{c} \\
\mathrm{kcal} / \mathrm{mol}\end{array}$ & atom & $X, \AA$ & $Y, \AA$ & $Z, \AA$ & charge $^{d}$ \\
\hline \multicolumn{9}{|c|}{ Neutral Molecules } \\
\hline \multirow[t]{2}{*}{$\mathrm{Tl}_{2} \mathrm{~T}_{2}$} & SP & -26.3891 & & $\begin{array}{r}\mathrm{Tl} \\
\mathrm{I}\end{array}$ & $\begin{array}{c} \pm 2.27 \\
0.0\end{array}$ & $\begin{array}{c}0.0 \\
\pm 2.31\end{array}$ & $\begin{array}{l}0.0 \\
0.052\end{array}$ & $\begin{array}{l}+0.61 \\
-0.61\end{array}$ \\
\hline & Td & no min & & & & & & \\
\hline \multirow[t]{3}{*}{$\mathrm{Pb}_{2} \mathrm{Te}_{2}$} & SP & -22.5722 & & $\mathrm{~Pb}$ & $\begin{array}{c} \pm 1.94 \\
0.0\end{array}$ & $\begin{array}{c}0.0 \\
+213\end{array}$ & $\begin{array}{l}0.0 \\
0.11\end{array}$ & $\begin{array}{l}+0.63 \\
-0.63\end{array}$ \\
\hline & $\mathrm{Td}$ & -22.5411 & +19.5 & $\mathrm{~Pb}$ & \pm 1.52 & 0.0 & 0.0 & +0.33 \\
\hline & & & & $\mathrm{Te}$ & 0.0 & \pm 1.39 & 2.25 & -0.33 \\
\hline \multirow{4}{*}{$\mathrm{Bi}_{2} \mathrm{Sb}_{2}$} & SP & -21.1069 & & $\mathrm{Bi}$ & \pm 1.63 & 0.0 & 0.0 & +0.34 \\
\hline & & & & $\mathrm{Sb}$ & 0.0 & \pm 2.14 & 0.85 & -0.34 \\
\hline & $\mathrm{Td}$ & -21.1779 & -44.6 & $\mathrm{Bi}$ & \pm 1.48 & 0.0 & 0.0 & +0.16 \\
\hline & & & & $\mathrm{Sb}$ & 0.0 & \pm 1.42 & 2.06 & -0.16 \\
\hline \multicolumn{9}{|c|}{ Anions } \\
\hline \multirow[t]{4}{*}{$\mathrm{Tl}_{2} \mathrm{Te}_{2}{ }^{2-}$} & SP & -19.6730 & & $\mathrm{Tl}$ & \pm 1.99 & 0.0 & 0.0 & +0.07 \\
\hline & & & & $\mathrm{Te}$ & 0.0 & \pm 2.32 & 0.04 & -1.07 \\
\hline & $\mathrm{Td}$ & -19.5964 & +48.1 & $\mathrm{Tl}$ & \pm 1.61 & 0.0 & 0.0 & -0.31 \\
\hline & & & & $\mathrm{Te}$ & 0.0 & \pm 1.41 & 2.63 & -0.69 \\
\hline \multirow{4}{*}{$\mathrm{Pb}_{2} \mathrm{Sb}_{2}{ }^{2-}$} & SP & -17.1874 & & $\mathrm{~Pb}$ & \pm 1.74 & 0.0 & 0.0 & -0.06 \\
\hline & & & & $\mathrm{Sb}$ & 0.0 & \pm 2.20 & 0.73 & -0.94 \\
\hline & $\mathrm{Td}$ & -17.2035 & -10.1 & $\mathrm{~Pb}$ & \pm 1.54 & 0.0 & 0.0 & -0.29 \\
\hline & & & & $\mathrm{Sb}$ & 0.0 & \pm 1.44 & 2.15 & -0.71 \\
\hline \multirow[t]{4}{*}{$\mathrm{Bi}_{2} \mathrm{Sn}_{2}{ }^{2-}$} & SP & -17.0809 & & $\mathrm{Bi}$ & \pm 2.22 & 0.0 & 0.0 & -0.75 \\
\hline & & & & $\mathrm{Sn}$ & 0.0 & \pm 1.72 & 0.71 & -0.25 \\
\hline & $\mathrm{Td}$ & -17.0991 & -11.4 & $\mathrm{Bi}$ & \pm 1.51 & 0.0 & 0.0 & -0.58 \\
\hline & & & & $\mathrm{Sn}$ & 0.0 & \pm 1.47 & 2.16 & -0.42 \\
\hline
\end{tabular}

${ }^{a}$ Results from closed-shell restricted Hartree-Fock calculations for the molecules listed. For the neutral molecules, the (4s $\left.4 \mathrm{p} 1 \mathrm{~d} / 3 \mathrm{~s} 3 \mathrm{p} 1 \mathrm{~d}\right)$ basis set was used. For the anions, the $(3 \mathrm{~s} 3 \mathrm{p} 1 \mathrm{~d} / 2 \mathrm{~s} 2 \mathrm{pld})$ basis set was used. ${ }^{b}$ Qualitative structure. SP indicates a square-planar-like structure; Td indicates a tetrahedral-like structure. 'Energy of the Td structure relative to the square-planar structure. ${ }^{d}$ Charge (based on the Mulliken population analysis) on the atoms of each type.

Table IV. Comparison of $X_{2} Y_{2}, 2 X Y$, and $2 X+2 Y$ Total Energies ${ }^{a}$

\begin{tabular}{lrcc}
\hline pair & $2 \mathrm{XY}$ & $\mathrm{X}_{2} \mathrm{Y}_{2}(\mathrm{SP})$ & $\mathrm{X}_{2} \mathrm{Y}_{2}(\mathrm{Td})$ \\
\hline $\mathrm{Tl}, \mathrm{I}$ & -129.3 & Neutral & \\
$\mathrm{Pb}, \mathrm{Te}$ & -84.0 & -144.4 & -94.9 \\
$\mathrm{Bi}, \mathrm{Sb}$ & +30.0 & +19.4 & -25.1 \\
& \multicolumn{3}{c}{ Anions } \\
$\mathrm{Tl}, \mathrm{Te}^{-}$ & -92.3 & -41.5 & +6.6 \\
$\mathrm{~Pb}^{-}, \mathrm{Sb}$ & -18.0 & +33.1 & +23.0 \\
$\mathrm{Bi}^{-} \mathrm{Sn}^{-}$ & -14.0 & +37.1 & +25.6 \\
\hline
\end{tabular}

${ }^{a}$ Energies in $\mathrm{kcal} / \mathrm{mol}$, relative to the total atomic energies in the appropriate basis set.

and electrons, the occupation yielding the lowest energy at the SP structures is $\left(4 a_{1}\right)^{2}\left(2 b_{1}\right)^{2}\left(2 b_{2}\right)^{2}\left(3 b_{2}\right)^{2}$. We denote this configuration as $3 b_{2}$ below. For the Td structures, the lowest energy orbital occupation is $\left(4 \mathrm{a}_{1}\right)^{2}\left(2 \mathrm{~b}_{1}\right)^{2}\left(2 \mathrm{~b}_{2}\right)^{2}\left(5 \mathrm{a}_{1}\right)^{2}$. This configuration is denoted as $5 a_{1}$ below. It is seen that even though these configurations are of the same symmetry $\left({ }^{1} \mathrm{~A}_{1}\right)$ they cannot smoothly interconvert (at the level of a single-configuration description) in $C_{2 v}$ symmetry because they have different orbital occupations. All these results are in excellent agreement with the abovementioned results concerning $\mathrm{Pb}_{2} \mathrm{Sb}_{2}{ }^{2-}, \mathrm{Sn}_{3} \mathrm{Bi}_{3}{ }^{2-}$ (both Td like), and $\mathrm{Tl}_{2} \mathrm{Te}_{2}{ }^{2-}$ (butterfly).

In Table IV, the total energies for the compounds of Table III are compared to the sum of the atomic energies of Table I. Where anions are concerned, we use the most stable atomic anion to calculate the relative energy. In addition, the energies of $2 X Y$ or $2 \mathrm{XY}^{-}$are presented for comparison, again relative to the sum of the atomic energies. It is seen that in the neutral molecules either geometry is stable to dissociation to atoms or $2 \mathrm{XY}$, except for $\mathrm{Bi}_{2} \mathrm{Sb}_{2}$, which is only more stable than separated atoms in its Td structure. In contrast, only one of the dianions is stable to dissociation to its constituent atoms, and in this case, it is still unstable to dissociation to $2 \mathrm{XY}^{-}$. In large measure, this is due to the double negative charges on these species. Assuming all the charge is located on the most electronegative elements, the purely electrostatic repulsion in $\mathrm{X}_{2} \mathrm{Y}_{2}{ }^{2-}$ is expected to be from 60 to 90 $\mathrm{kcal}$, depending on the geometry. This will, to a large extent, be compensated for by the presence of counterions. Thus, it is quite
Table V. Energy versus Reaction Parameter for Single Configuration SCF's $\mathrm{Pb}_{2} \mathrm{Te}_{2}{ }^{a}$

\begin{tabular}{|c|c|c|c|c|c|}
\hline \multirow[b]{2}{*}{$q$} & \multicolumn{2}{|c|}{$\Delta E, \mathrm{kcal} / \mathrm{mol}$} & \multirow[b]{2}{*}{$q$} & \multicolumn{2}{|c|}{$\Delta E, \mathrm{kcal} / \mathrm{mol}$} \\
\hline & $5 a_{1}$ & $3 b_{2}$ & & $5 a_{1}$ & $3 b_{2}$ \\
\hline 0.0 & 0.0 & 116.5 & 0.6 & 67.2 & 0.5 \\
\hline 0.1 & 3.7 & 94.0 & 0.7 & 84.9 & -8.2 \\
\hline 0.2 & 12.3 & 65.9 & 0.8 & 105.4 & -14.6 \\
\hline 0.3 & 23.7 & 43.7 & 0.9 & 130.7 & -18.5 \\
\hline 0.4 & 36.8 & 26.0 & 1.0 & 170.0 & -19.5 \\
\hline 0.5 & 51.3 & 11.8 & & & \\
\hline
\end{tabular}

${ }^{a}$ The energies are relative to the one-configuration $5 a_{1}$ SCF at $q=$ 0.0 ( $E=-22.5412$ hartrees). The column marked $5 \mathrm{a}_{1}$ gives the energies for the $5 a_{1}$ configuration; the column marked $3 b_{2}$ gives the energies for the $3 b_{2}$ configuration.

possible that the $\mathrm{X}_{2} \mathrm{Y}_{2}{ }^{2-}$ compounds would be stable, relative to separated atoms, in the presence of counterions. The reason that the dianions do not dissociate to separated atoms in the geometry optimization is that we have assumed an orbital occupation that corresponds to a closed-shell singlet wave function. In order for this occupation to dissociate to separated atoms, it would have to go to highly excited atoms. As a result, we find a $C_{2 v}$ minimum, even though it is above the separated atoms limit. It should be noted that even the neutral species are biased toward the separated atom limit, since the correlation energy is expected to be greatest for the molecules and we have neglected electron correlation in all cases.

Since we have found two stable minima, $\mathrm{Td}$ and $\mathrm{SP}$, for $\mathrm{Pb}_{2} \mathrm{Te}_{2}$, we need to know if an energy barrier separates the two minima. For that reason, we present in Table $\mathrm{V}$ the results from singleconfigurational SCF calculations on the compound $\mathrm{Pb}_{2} \mathrm{Te}_{2}$. The SCF results are for the two configurations $5 \mathrm{a}_{1}$ and $3 \mathrm{~b}_{2}$, which are those appropriate for the description of the ground state at the two $C_{2 v}$ stationary points The results in Table $\mathrm{V}$ are based on the assumption of a least-motion path between the two minima. The parameter $q$ measures the fraction of the distance traveled along this assumed reaction path, with $q=0$ corresponding to the $T d$ structure. A number of interesting points can be observed. In $C_{2 v}$ symmetry, there is a crossing between these two configurations at approximately $q=0.4$. In addition, the $5 a_{1}$ orbital is localized 
Table VI. Open-Shell Singlet and Triplet States for Neutral $\mathrm{X}_{2} \mathrm{Y}_{2}$ Molecules ${ }^{\circ}$

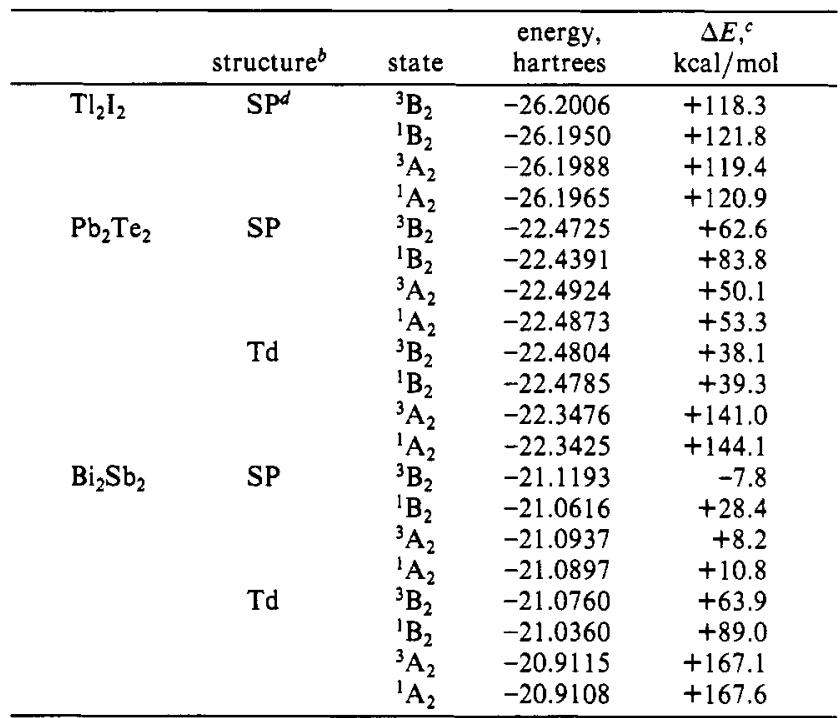

${ }^{a}$ Results from open-shell restricted Hartree-Fock calculations for the molecules listed. The $(4 \mathrm{~s} 4 \mathrm{pld} / 3 \mathrm{~s} 3 \mathrm{pld})$ basis set was used. ${ }^{b}$ Qualitative structure for the closed-shell $C_{2}$ minimum from Table III. ' Energy of the given state relative to the closed-shell SCF ground state at the given geometry. ${ }^{d}$ Only the square-planar structure was considered for $\mathrm{Tl}_{2} \mathrm{I}_{2}$.

on $\mathrm{Pb}$ and the $3 \mathrm{~b}_{2}$ orbital is localized on $\mathrm{Te}$. Thus, one expects a weak coupling matrix element between these configurations. Therefore, is is expected that in $C_{2 v}$ symmetry a barrier should persist between the two local minima even with a multiconfigurational description of the wave function and that the character of the lowest state should change rather abruptly with $q$ in the region around $q=0.4$.

In Table VI, we present single-point calculations for open-shell triplet and singlet states for the three neutral molecules at both the Td and SP closed-shell optimum geometries. The electronic occupations are those of the ground state except that in place of the doubly occupied $3 b_{2}$ or $5 a_{1}$ orbital of the ground state (depending on the geometry) we have the occupation $5 a_{1}{ }^{1} 3 b_{2}{ }^{1}$ for the $B_{2}$ states and $3 b_{2}{ }^{1} 3 b_{1}{ }^{1}$ for the $A_{2}$ states. These results can be used to provide a qualitative idea of the $5 a_{1}-3 b_{2}$, orbital energy gaps. It is seen that for both geometries of $\mathrm{Pb}_{2} \mathrm{Te}_{2}$ the closed-shell singlet is the lowest energy state. This is also the case for the $T_{d}$ geometry of $\mathrm{Bi}_{2} \mathrm{Sb}_{2}$ and the square-planar geometry of $\mathrm{Tl}_{2} \mathrm{I}_{2}$. However, the closed-shell singlet state of $\mathrm{Bi}_{2} \mathrm{Sb}_{2}$ at the squareplanar geometry is actually above one of the triplet states, and both of the open-shell singlets are quite close in energy to the closed-shell state. In all cases, the open-shell singlet is higher than the triplet, as it should be at the SCF level when the open-shell electrons occupy orthogonal orbitals. In $C_{2 v}$ symmetry, the open-shell and closed-shell states cannot interact, since the former are of ${ }^{1} B_{2}$ symmetry, while the latter are of ${ }^{1} A_{1}$ symmetry. However, their energetic proximity at the higher energy $C_{2 v}$ minima suggests that second-order Jahn-Teller interactions might be important. (Further evidence for this is presented in Table VII.) In these cases, use of multiconfigurational wave functions in symmetries lower than $C_{2 v}$ would be useful in examining the potential surface.

Finally, in Table VII we present the results from geometry optimizations for the neutral $\mathrm{X}_{2} \mathrm{Y}_{2}$ compounds using the (3s $3 \mathrm{p} / 2 \mathrm{~s} 2 \mathrm{p}$ ) basis sets in both $C_{2 v}$ and $C_{1}$ symmetry. Our aim in these calculations was to explore the stability of the $C_{2 v}$ structures to symmetry lowering; thus, we compare the results from optimizations performed in $C_{2 v}$ and $C_{1}$ symmetries for $\mathrm{Pb}_{2} \mathrm{Te}_{2}, \mathrm{Bi}_{2} \mathrm{Sb}_{2}$, and $\mathrm{Tl}_{2} \mathrm{I}_{2}$. As before, we sought both $\mathrm{Td}$ and $\mathrm{SP}$ stationary points. Comparing the $C_{2 v}$ results in this basis to those in the larger basis, it is seen that the total energies are somewhat higher $(\sim 30-100 \mathrm{mh})$ than those from the larger basis set used in the results of tables I-V. The geometries are also somewhat different from those obtained in the larger basis. In general, the internuclear separations are smaller in the larger basis, by up to $0.15 \AA$. In addition, the energy differences between the Td and SP structures tend to be biased toward the SP structure, relative to the larger basis results. However, the same trend is found in the smaller basis set, and the energy differences are at the least qualitatively correct. Thus, we have used this smaller basis to assess the stability of the compounds to distortions that lower the symmetry from $C_{2 v}$.

In the first calculations, labeled $C_{1} A$, we began the geometry optimizations near the $C_{2}$, structure but with small distortions from it to yield an initial structure of overall $C_{1}$ symmetry. The ge-

Table VII. Neutral Closed-Shell $\mathrm{X}_{2} \mathrm{Y}_{2}$ Molecules in $(3 \mathrm{~s} 3 \mathrm{p} / 2 \mathrm{~s} 2 \mathrm{p})$ Basis Set $^{a}$

\begin{tabular}{|c|c|c|c|c|c|c|c|c|}
\hline & structure $\mathrm{e}^{b}$ & $\begin{array}{l}\text { energy, } \\
\text { hartrees }\end{array}$ & symmetry $^{d}$ & $\begin{array}{c}\Delta E^{\mathrm{c}} \\
\mathrm{kcal} / \mathrm{mol}\end{array}$ & atom & $X, \AA$ & $Y, \AA$ & $Z, \AA$ \\
\hline & & & Neutr & lecules & & & & \\
\hline \multirow[t]{2}{*}{$\mathrm{Tl}_{2} \mathrm{I}_{2}$} & SP & -26.3696 & $C_{2 v}$ & & $\mathrm{Tl}$ & \pm 2.40 & 0.0 & 0.0 \\
\hline & & $\begin{array}{l}-26.3696^{\text {ef }} \\
-26.3696^{\text {ef }}\end{array}$ & $\begin{array}{l}\mathrm{C}_{1} \mathrm{~A} \\
\mathrm{C}_{1} \mathrm{~B}\end{array}$ & & I & 0.0 & \pm 2.21 & 0.11 \\
\hline \multirow[t]{4}{*}{$\mathrm{Pb}_{2} \mathrm{Te}_{2}$} & SP & -22.5164 & $C_{2 v}$ & & $\begin{array}{l}\mathrm{Pb} \\
\mathrm{Te}\end{array}$ & $\begin{array}{c} \pm 2.05 \\
0.0\end{array}$ & $\begin{array}{c}0.0 \\
\pm 2.08\end{array}$ & $\begin{array}{l}0.0 \\
0.12\end{array}$ \\
\hline & & $\begin{array}{l}-22.5164^{e} \\
-22.5164^{e}\end{array}$ & $\begin{array}{l}\mathrm{C}_{1} \mathrm{~A} \\
\mathrm{C}_{1} \mathrm{~B}\end{array}$ & & & & & \\
\hline & $\mathrm{Td}$ & -22.4690 & $C_{2 v}$ & +29.7 & $\begin{array}{l}\mathrm{Pb} \\
\mathrm{Te}\end{array}$ & $\begin{array}{c} \pm 1.55 \\
0.0\end{array}$ & $\begin{array}{c}0.0 \\
\pm 1.45\end{array}$ & $\begin{array}{l}0.0 \\
2.32\end{array}$ \\
\hline & & $\begin{array}{l}-22.4690^{\circ} \\
-22.5160^{8}\end{array}$ & $\begin{array}{l}\mathrm{C}_{1} \mathrm{~A} \\
\mathrm{C}_{1} \mathrm{~B}\end{array}$ & & & & & \\
\hline \multirow[t]{4}{*}{$\mathrm{Bi}_{2} \mathrm{Sb}_{2}$} & SP & -21.0338 & $C_{2 v}$ & & $\begin{array}{l}\mathrm{Bi} \\
\mathrm{Sb}\end{array}$ & $\begin{array}{c} \pm 1.68 \\
0.0\end{array}$ & $\begin{array}{c}0.0 \\
\pm 2.16\end{array}$ & $\begin{array}{l}0.0 \\
0.87\end{array}$ \\
\hline & & $\begin{array}{l}-21.0338^{e} \\
-21.0368^{h}\end{array}$ & $\begin{array}{l}\mathrm{C}_{1} \mathrm{~A} \\
\mathrm{C}_{1} \mathrm{~B}\end{array}$ & & & & & \\
\hline & $T d$ & -21.0840 & $C_{2 v}$ & -31.5 & $\begin{array}{l}\mathrm{Bi} \\
\mathrm{Sb}\end{array}$ & $\begin{array}{c} \pm 1.51 \\
0.0\end{array}$ & $\begin{array}{c}0.0 \\
\pm 1.47\end{array}$ & $\begin{array}{l}0.0 \\
2.11\end{array}$ \\
\hline & & $\begin{array}{c}-21.0840^{e} \\
i\end{array}$ & $\begin{array}{l}\mathrm{C}_{1} \mathrm{~A} \\
\mathrm{C}_{1} \mathrm{~B}\end{array}$ & & & & & \\
\hline
\end{tabular}

${ }^{a}$ Results from closed-shell restricted Hartree-Fock calculations for the molecules listed. ${ }^{b}$ Qualitative structure for the $\mathrm{C}_{2 v}$ geometry optimization. ${ }^{c}$ Energy of the Td structure relative to the square-planar structure. ${ }^{d}$ Symmetry in which the geometry optimization was performed. See Computational Results section for definitions of the A- and B-type $C_{1}$ optimizations. 'The internuclear distances for the $C_{1}$ structures differ from the $C_{20}$ structures by at most $0.03 \AA$. ${ }^{f}$ These structures had two TlI bonds of $3.25 \AA$ and two of $3.29 \AA$, whereas the $C_{2 v}$ structure in this basis had four equal bonds of $3.26 \AA .{ }^{g}$ From this distorted geometry, the optimization converged to the SP geometry. ${ }^{h}$ The structure obtained was rectangularly distorted, having two short BiSb bonds $(2.73 \AA)$ and two long BiSb bonds $(3.05 \AA)$ whereas the $C_{2 v}$ structure had all four BiSb bonds equal at 2.87 $\AA$ in this basis. 'The SCF did not converge at the distorted structure. 
ometry obtained from this starting point is essentially the $C_{2 v^{-}}$ constrained geometry in all cases. Thus, the $C_{2 v}$ structures are stable to small symmetry-breaking distortions; i.e., they are true local minima.

We also sought other local minima. As will be discussed below, the isovalent compound cyclobutadiene is prone to rectangular distortions, rather than the rhomboidal distortions allowed for the $\mathrm{X}_{2} \mathrm{Y}_{2}$ compounds in $C_{2 v}$ symmetry. We thus began the geometry optimizations labeled $\mathrm{C}_{1} \mathrm{~B}$ in a rectangularly distorted geometry. It is seen that, for the single-configurational closed-shell wave functions considered here, the $C_{2 v}$ minima are unstable with respect to large distortions for three cases. For the large distortions examined around the $\mathrm{Pb}_{2} \mathrm{Te}_{2} \mathrm{Td}$ minimum, the optimization converged to the SP structure. Smaller distortions might have converged to the $\mathrm{Td}$ point, as was the case for the A-type distortions, but in any event, the minimum does not appear to be very broad. $\mathrm{Bi}_{2} \mathrm{Sb}_{2}$, in the vicinity of its square-planar $C_{2 v}$ minimum, distorts to yield a structure having two short and two long $\mathrm{BiSb}$ bonds. $\mathrm{Tl}_{2} \mathrm{I}_{2}$ also yields a slightly distorted structure when $C_{2 v}$ symmetry is relaxed, leading to a structure having two TlI bonds of length $3.25 \AA$ and two TlI bonds of length $3.29 \AA$. The $C_{20}$ structure in this basis has four equivalent $3.26-\AA$ bonds. However, the energy of the distorted structure is identical with the $C_{2 v}$ structure to $\pm 1.0 \mathrm{kcal} / \mathrm{mol}$. The surface is apparently relatively flat in this region, and without enlarging the basis and addition of correlation, it is not possible to assess whether this distortion is real or not. Thus, only for $\mathrm{SP} \mathrm{Bi}_{2} \mathrm{Sb}_{2}$ do we obtain evidence that rectangular distortions, of the type important for cyclobutadiene, are important. However, the energy difference between the $C_{2 v}$ and the distorted structures is small and could be altered in a larger basis or with addition of correlation.

It should be noted that, even though $\mathrm{Bi}_{2} \mathrm{Sb}_{2}$ in the square-planar geometry is higher energy than separated atoms (see Table II), the $C_{1}$ geometry optimization does not yield separated atoms. As was the case for the dianions, the calculation assumed the molecule is a closed-shell singlet, and dissociation to separated atoms as closed-shell singlet yields an excited state.

2. Discussion. The calculations presented above provide a number of interesting results regarding the geometries and energetics of these models of the Zintl anions. For both the neutral and the dianionic species, we obtain evidence for Td and SP geometries; in most cases, two local minima exist. There is also a clear trend for preferring square-planar structures when the electronegativity difference between $\mathrm{X}$ and $\mathrm{Y}$ increases. In addition, the SP structures flatten considerably with increasing the electronegativity difference between $X$ and $Y$. These results substantiate the qualitative analysis of section III. In most cases, the neutral $\mathrm{X}_{2} \mathrm{Y}_{2}$ molecules are stable with respect to dissociation to $2 \mathrm{XY}$ or $2 \mathrm{X}+2 \mathrm{Y}$, exceptions being square-planar $\mathrm{Bi}_{2} \mathrm{Sb}_{2}$ and $\mathrm{Td} \mathrm{Tl}_{2} \mathrm{I}_{2}$ (where the $3 \mathrm{~b}_{2}$ configuration is lowest, even in a $\mathrm{Td}$ geometry, thus yielding the square-planar structure from whatever point the optimization was begun).
It is interesting to remark that, from our calculations for the SP $A_{2}$ excited states (Table VI), the energy difference with respect o the ground state significantly increases as the $\mathrm{X}-\mathrm{Y}$ electroregativity difference increases. This is the result of the opening If an energy gap between $3 b_{1}$ and $3 b_{2}$ orbitals near the SP gemetry as anticipated in the qualitative description in section III. These results also show that, for large $X-Y$ electronegativity differences, the 20-electron butterfly cluster tends to collapse into a SP-type structure. Nevertheless, as was suggested by the results of Table III, this collapse is a sensitive function of the electronegativity difference. We thus anticipate that butterfly clusters with relativity small $\theta$ values could be found.

\section{Conclusions}

The correlation diagram for the tetrahedral $\left(T_{d}\right) \rightarrow$ butterfly $\left(C_{2 v}\right) \rightarrow$ square planar $\left(D_{2 h}\right)$ transformation of tetranuclear main group clusters suggests the possibility of two different electron counts, 20 and 22 electrons, for the butterfly clusters. The lower electron count is also the one appropriate for tetrahedral clusters. The analysis also shows that the transformation tetrahedral $\rightarrow$ butterfly is a symmetry-forbidden process for 20 electrons. As a result, clusters with this electron count could be found in two different geometries. In addition, the analysis also shows that the butterfly structure becomes favored in $X_{2} Y_{2}$ clusters where the two atoms $X$ and $Y$ are of different electronegativity.

$\mathrm{Ab}$ initio calculations give evidence for two distinct minima in the several neutral $X_{2} Y_{2}$ compounds studied that have been used to model the Zintl anions. Symmetry-constrained calculations on the Zintl dianions also yield two distinct local $C_{2 v}$ stationary points, but because of the absence of counterions, the dianions are not stable with respect to dissociation to separated atoms.

It was found that for the neutral species the ground state in all but one case is clearly a closed-shell singlet, at least at the SCF level. The change in the most stable structure was analyzed on the basis of an orbital correlation diagram for conversion between the Td and square-planar local minima. It was seen that the change in the most stable electronic configuration is caused by a crossing of a $5 \mathrm{a}_{1}$ orbital and a $3 \mathrm{~b}_{2}$ orbital. Electronegativity differences between $X$ and $Y$ adjust the difference in energy between these two orbitals and thereby affect which of the two local minima is the overall lowest. These results are in agreement with the fact that the clusters $\mathrm{Pb}_{2} \mathrm{Sb}_{2}{ }^{2-}$ and $\mathrm{Sb}_{2} \mathrm{Bi}_{2}{ }^{2-}$ have a tetrahedral-like geometry, while $\mathrm{Tl}_{2} \mathrm{Te}_{2}{ }^{2-}$ is a butterfly cluster.

Acknowledgment. O.E. is grateful to the Indiana University Institute for Advanced Study for providing financial support for her stay in 1988. E.R.D. and O.E. thank the NSF and CNRS for an international travel grant. The ab initio calculations were carried out by R.C. and E.R.D. with NSF grant support.

Registry No. $\mathrm{Tl}_{2} \mathrm{I}_{2}, 7790-30-9 ; \mathrm{Pb}_{2} \mathrm{Te}_{2}, 122521-48-6 ; \mathrm{Bi}_{2} \mathrm{Sb}_{2}, 12323-$ 19-2; $\mathrm{Tl}_{2} \mathrm{Te}_{2}{ }^{2-}, 77321-80-3 ; \mathrm{Pb}_{2} \mathrm{Sb}_{2}{ }^{2-}, 113273-58-8 ; \mathrm{Bi}_{2} \mathrm{Sn}_{2}{ }^{2-}, 113251-$ $42-6$. 